\title{
Hydrothermal Behavior of Transient Fluid Flow and Heat Transfer Through a Rotating Curved Rectangular Duct with Natural and Forced Convection
}

\author{
Ratan Kumar Chanda ${ }^{1}$, Mohammad Sanjeed Hasan², Md. Mahmud Alam ${ }^{3}$, Rabindra Nath Mondal ${ }^{*}$ \\ ${ }^{1}$ Department of Mathematics, Jagannath University, Dhaka 1100, Bangladesh \\ ${ }^{2}$ Department of Mathematics, Bangabandhu Sheikh Mujibur Rahman Science and Technology University, Gopalganj 8100, \\ Bangladesh \\ ${ }^{3}$ Mathematics Discipline, Khulna University, Khulna 9208, Bangladesh
}

Corresponding Author Email: rnmondal@jnu.ac.bd

https://doi.org/10.18280/mmep.070401

Received: 20 July 2020

Accepted: 15 December 2020

\section{Keywords:}

rotating curved duct, Taylor number, secondary

flow, isotherm, time-progression

\begin{abstract}
The present work explores a spectral-based computational study on hydrothermal behavior of transient fluid flow with natural and forced convective heat transfer through a rotating curved rectangular duct of strong curvature. The outer wall of the duct is heated while the inner wall cooled, the other walls being thermally insulated. The system rotates about the vertical axis in the positive and negative direction for the Taylor number $(-1000 \leq \operatorname{Tr} \leq 1000)$ with a constant non-dimensional pressure gradient force, the Dean number Dn $=2000$. Time-history analysis is performed and fluid characteristics are well determined by depicting the phase space of the time-history result. It is found that the chaotic flow turns into steady-state flow through multiperiodic and periodic oscillating flows, if $\mathrm{Tr}$ is increased either in the positive or in the negative direction. Streamlines of secondary flow and isotherms are obtained at some specific values of $\mathrm{Tr}$, and it is found that the time-dependent flow consists of asymmetric 2- to multi-vortex solutions. Vortex structure of secondary flows is obtained for physically realizable solutions and it is found that maximum 8-vortex is obtained for the chaotic solution while 2-vortex for the steady-state solution. Nusselt number as well as temperature gradient is calculated as an index of heat transfer, and it is found that convective heat transfer is significantly enhanced by the secondary flow; and the chaotic flow, which occurs relatively at small $\mathrm{Tr}$, boosts heat transfer more effectively than the steady-state or other solutions. Finally, our numerical results have been validated with the experimental outcomes and it is found that there is a good agreement between the numerical and experimental investigations.
\end{abstract}

\section{INTRODUCTION}

Due to ample applications from medical services to industrial activities, the study of flow and heat transfer through a curved duct has attracted considerable attention to the researchers from the past to the present. When a fluid flows in a curved duct, the flow experiences helical streamlines. This is called secondary flow, which is a relatively minor flow imposed on the primary flow. The secondary flow is found in numerous flow situations such as in the blade passage in turbomachinery, a bent pipe, internal combustion engines, aircraft intakes, refrigeration, air conditioning systems, diffusers and heat exchangers, not to mention applications in other areas such as blood flow in human and other animals. The magnitude of these secondary flows is quantified by a nondimensional parameter, the Dean number, $D n$, defined by, $D n=R e \sqrt{\frac{D_{h}}{2 R}}$, where $R e$ is the Reynolds number, $D_{h}$ is the hydraulic diameter and $R$ is the radius of curvature of the curved channel. When fluid is flowing through a curved channel, centrifugal force takes into effect and the flow velocity closer to the outer wall is greater than the flow velocity at the inner wall. As the fluid near the outer wall moves with relatively large velocity, an apparent vacuum at the inner wall is generated immediately in front of the flow and the fluid closer to the outer wall shows a tendency to move towards the inner wall. This circulatory flow from the outer curved wall to the inner wall is named as Dean vortices in recognition of the pioneer work by Dean [1]. After that, a significant number of theoretical and experimental investigations have been made; here the articles by Berger et al. [2], Nandakumar and Masliyah [3], Ito [4], Yanase et al. [5] and Mondal [6] may be referenced.

It is worth mentioning that the fluid flowing in a rotating curved duct is subjected to two forces: the Coriolis force and the centrifugal force. Due to combined action of these two forces a complex behavior of the secondary flow and the axial flow is obtained, and when a temperature induced variation of fluid density occurs for non-isothermal flows, both Coriolis and centrifugal forces contribute to the generation of vorticity [7]. Ghia and Shokhey [8] showed that the duct aspect ratio and curvature ratio significantly influence Dean instability in the curved channel. Ishigaki [9] examined the flow structure and friction factor for both counter-rotating and co-rotating curved pipe with small curvature. Selmi et al. [10] examined combined effects of system rotation and curvature on the bifurcation structure of two-dimensional flows in a rotating curved square duct. Wang and Cheng [11] examined the flow 
characteristics and heat transfer in curved square ducts for positive rotation and found reverse secondary flow for the corotation cases. Recently, Mondal et al. [12] predicted a numerical study on curved rectangular duct flow and compared their results with the experimental data obtained by Chandratilleke et al. [13] and visualized the stream functions and the isotherms for different Dean numbers and the Grashof number. Norouzi et al. [14] elaborated first and second normal stresses with enumerating the Nusselt number and the influence on secondary flow intensity in the elastic property. For curved rectangular duct, Chandratilleke et al. [15] reported an extensive parametric study, capturing the profound influence from duct aspect ratio, curvature ratio and wall heat flux on the flow behavior. Recently, Hasan et al. [16] and Hasan et al. [17] employed spectral method to study steady solutions and unsteady flow characteristics through a rotating and non-rotating curved square duct for constant curvature, and showed combined effects of centrifugal-Coriolisbuoyancy forces on fluid flows in the curved channel.

With a view to study non-linear behavior of the unsteady solutions, Yanase and Nishiyama [18] first initiated timedependent solutions through a curved rectangular duct. Wang and Yang [19] performed numerical as well as experimental investigation on fully developed periodic oscillation in a curved square duct. Mondal et al. [20] performed numerical prediction of isothermal flow with unsteady solutions through a stationary curved square duct, where they showed that periodic solution turns into chaotic solution via multi-periodic solution, if the Dean number is increased. The secondary flow characteristics in a curved square duct were investigated experimentally by using visualization method by Yamamoto et al. [21]. Mondal et al. [22] performed numerical prediction of non-isothermal flow with unsteady solutions through a stationary curved square duct, where they showed that periodic solutions turn into chaos through a multi-periodic solution, if the Dean number is increased no matter what the curvature is. Time evolution calculation with the variation of centrifugal and Coriolis instability has been predicted by Mondal et al. [23] at a high-pressure gradient over a wide range of the Taylor number. Unsteady flow characteristics, heat transfer and entropy generation has been performed by Razavi et al. [24]. For solving the unsteady flow through a curved tube with strong curvature, recently Krishna et al. [25] performed flow visualization and computational fluid dynamics. Recently, Islam et al. [26] showed transitional behavior of the flow in a rotating curved duct for various Dean numbers over a wide range of the Taylor number. Very recently, Hasan et al. [27], Hasan et al. [28] and Hasan et al. [29] studied unsteady behavior of the flow through a rotating curved square duct for constant Dean number. However, hydro-thermal behavior of the unsteady solution is not yet resolved, in detail, for the flow through a rotating curved rectangular duct with strong curvature to predict the influence of centrifugal and Coriolis forces on fluid flows, which motivated us to do this study to fill up this gap.

To study heat transfer through curved channels, Chandratilleke [30] reported experimental results for the flow in curved rectangular duct with external heating of the outer wall. He showed that convective heat transfer is significantly enhanced by the secondary flow particularly when the Dean vortices emerge at the outer wall. Yanase et al. [31] and Mondal et al. [22] studied time-dependent behavior of the unsteady solutions for curved rectangular/square duct flow and showed that secondary flows enhance heat transfer in the flow. Norouzi et al. [32] investigated fully developed flow and heat transfer of viscoelastic materials in curved square ducts under constant heat flux. Chandratilleke et al. [13] presented extensive 3D computational study using helicity function that describes the secondary vortex structure and thermal behavior in the fluid flow through curved rectangular ducts of aspect ratios ranging from 1 to 6 . Norouzi and Biglari [33] performed, for the first time, an analytical solution of Dean flow inside a curved rectangular duct by using perturbation method. Their study was limited to low Reynolds numbers and obtained maximum four-vortex solutions. $\mathrm{Wu}$ et al. [34] performed numerical study of the secondary flow characteristics in a curved square duct by using spectral method, where the walls of the duct except the outer wall rotate around the centre of curvature and an azimuthal pressure gradient was imposed. Recently, Hasan et al. $[35,36]$ undertook numerical prediction of fluid flow and heat transfer through a non-rotating curved square channel for various Dean and Grashof numbers. The study elucidated role of secondary vortices on convective heat transfer which showed that convective heat transfer is significantly enhanced by the secondary flow; and the chaotic flow, which takes place at large Dean and Grashof numbers, enhances heat transfer more efficiently. Very recently, Ray et al. [37] applied spectral method to predict hydrodynamic instability and convective heat transfer through a rotating curved rectangular duct of moderate curvature. They obtained three branches of steady solutions and showed that chaotic flow turns into steady-state flow through periodic oscillating flow, if $\mathrm{Tr}$ is increased in the positive direction. As par to the authors' knowledge, however, there has not been done any substantial work studying the hydro-thermal behavior of fluid flow with forced and natural convective heat transfer through a rotating curved rectangular channel in the presence of strong centrifugal force for both positive and negative rotation of the channel. But from the scientific as well as engineering point of view it is quite interesting to study this type of flow because it is often encountered in engineering applications such as in gas turbine, plastic industry and metallic industry.

In the ongoing paper, our intention is to study hydro-thermal behavior with flow transition through a rotating curved rectangular duct of strong curvature. Studying the vortex structure as well as effects of rotation on the unsteady flow characteristics, caused by the combined action of the centrifugal, Coriolis and buoyancy forces, is an important objective of the present study.

\section{MATHEMATICAL MODEL AND GOVERNING EQUATIONS}

Consideration is given for hydro-dynamically and thermally fully developed two-dimensional laminar flow of viscous incompressible fluid streaming through a rotating curved rectangular duct of aspect ratio 2 and curvature ratio 0.5 . The system rotates at a variable angular velocity $\Omega_{T}$ around the $y^{\prime}$ axis. It is assumed that the outer wall of the duct is heated while the inner wall cooled, the top and bottom walls being thermally insulated. The $x$-, $y$ - and $z$-axes are taken to be in the horizontal, vertical and axial directions respectively. It is assumed that the flow is driven by a as shown in Figure 1. 


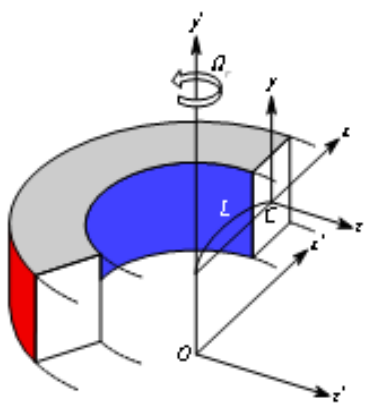

(a)

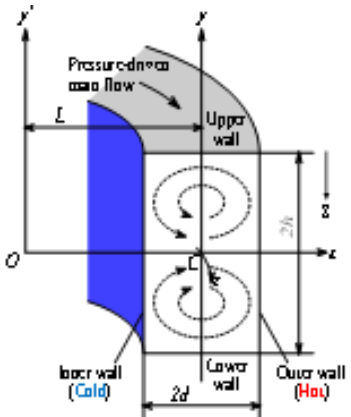

(b)
Figure 1. (a) Coordinate system, (b) Cross section of the curved duct

The dimensional variables are non-dimensionalized by using the representative length $d$ and the representative velocity $U_{0}=\frac{v}{d}$, where $v$ is the kinematic viscosity of the fluid. The stream functions for cross-sectional velocities have the following form:

$$
\left.\begin{array}{l}
u=\frac{1}{r} \frac{\partial \psi}{\partial y}=\frac{1}{1+\delta x} \frac{\partial \psi}{\partial y} \\
v=\frac{1}{r} \frac{\partial \psi}{\partial x}=-\frac{1}{1+\delta x} \frac{\partial \psi}{\partial x}
\end{array}\right\}
$$

Now, stream-wise velocity (w), cross-sectional streamfunction $(\psi)$ and temperature profile $(T)$ are derived based on the Navier-Stokes equation and energy equation as follows:

$$
\begin{aligned}
& (1+\delta x) \frac{\partial w}{\partial t}=D n-\frac{\partial(w, \psi)}{\partial(x, y)}+\frac{\delta^{2} w}{1+\delta x}+(1+\delta x) \Delta_{2} w- \\
& \frac{\delta}{1+\delta x} \frac{\partial \psi}{\partial y} w+\delta \frac{\partial w}{\partial x}-\delta \operatorname{Tr} \frac{\partial \psi}{\partial y} \\
& \frac{\partial T}{\partial t}+\frac{1}{(1+\delta x)} \frac{\partial(T, \psi)}{\partial(x, y)}=\frac{1}{\operatorname{Pr}}\left(\Delta_{2} T+\frac{\delta}{1+\delta x} \frac{\partial T}{\partial x}\right) \\
& \left(\Delta_{2}-\frac{\delta}{1+\delta x} \frac{\partial}{\partial x}\right) \frac{\partial \psi}{\partial t}=-\frac{1}{(1+\delta x)} \frac{\partial\left(\Delta_{2} \psi, \psi\right)}{\partial(x, y)} \\
& +\frac{\delta}{(1+\delta x)^{2}}\left[\frac{\partial \psi}{\partial y}\left(2 \Delta_{2} \psi-\frac{3 \delta}{1+\delta x} \frac{\partial \psi}{\partial x}+\frac{\partial^{2} \psi}{\partial x^{2}}\right)-\frac{\partial \psi}{\partial x} \frac{\partial^{2} \psi}{\partial x \partial y}\right] \\
& +\frac{\delta}{(1+\delta x)^{2}} \times\left[3 \delta \frac{\partial^{2} \psi}{\partial x^{2}}-\frac{3 \delta^{2}}{1+\delta x} \frac{\partial \psi}{\partial x}\right]-\frac{2 \delta}{1+\delta x} \frac{\partial}{\partial x} \Delta_{2} \psi \\
& +w \frac{\partial w}{\partial y}+\Delta_{2}^{2} \psi-G r(1+\delta x) \frac{\partial T}{\partial x}+\frac{1}{2} \operatorname{Tr} \frac{\partial w}{\partial y}
\end{aligned}
$$

where,

$$
\Delta_{2} \equiv \frac{\partial^{2}}{\partial x^{2}}+\frac{1}{4} \frac{\partial^{2}}{\partial y^{2}}, \frac{\partial(f, g)}{\partial(x, y)} \equiv \frac{\partial f}{\partial x} \frac{\partial g}{\partial y}-\frac{\partial f}{\partial y} \frac{\partial g}{\partial x}
$$

In Eqns. (2)-(4), four non-dimensional parameters $D n, G r$, $P r$ and $T r$ are used which are defined as:

$$
D n=\frac{G d^{3}}{\mu v} \sqrt{\frac{2 d}{L}}, G r=\frac{\beta g \Delta T d^{3}}{v^{2}}, \operatorname{Pr}=\frac{v}{\kappa}, T r=\frac{2 \sqrt{2 \delta} \Omega d^{3}}{v \delta}
$$

The applied boundary conditions for $w$ and $\psi$ are used as:

$$
\left.\begin{array}{r}
w( \pm 1, y)=w(x, \pm 1)=\psi( \pm 1, y)=\psi(x, \pm 1)=0 \\
\frac{\partial \psi}{\partial x}( \pm 1, y)=\frac{\partial \psi}{\partial y}(x, \pm 1)=0
\end{array}\right\}
$$

and temperature $T$ is assumed to be constant on the walls as:

$$
T(1, y)=1, \quad T(-1, y)=-1, \quad T(x, \pm 1)=x
$$

In the present study, $\operatorname{Tr}$ varies in both positive and negative, while $D n, G r, \delta$ and $P r$ are fixed as $D n=2000, G r=100$, $\delta=0.5$ and $\operatorname{Pr}=7.0$ (water)

\section{NUMERICAL COMPUTATION}

\subsection{Procedure to numerical calculation}

We applied spectral method to solve the Eqns. (2) to (4) numerically. By this method the variables are expanded in a series of functions consisting of Chebyshev polynomials. That is, the expansion functions $\varphi_{n}(x)$ and $\psi_{n}(x)$ are expressed as:

$$
\left.\begin{array}{l}
\varphi_{n}(x)=\left(1-x^{2}\right) \quad C_{n}(x), \\
\psi_{n}(x)=\left(1-x^{2}\right)^{2} \quad C_{n}(x)
\end{array}\right\}
$$

where, $C_{n}(x)=\cos \left(n \cos ^{-1}(x)\right)$ is the $n^{\text {th }}$ order Chebyshev polynomial. $w(x, y, t), \psi(x, y, t)$ and $T(x, y, t)$ are expanded in terms of the expansion functions $\varphi_{n}(x)$ and $\psi_{n}(x)$ as:

$$
\left.\begin{array}{c}
w(x, y, t)=\sum_{m=0}^{M} \sum_{n=0}^{N} w_{m n}(t) \phi_{m}(x) \phi_{n}(y) \\
\psi(x, y, t)=\sum_{m=0}^{M} \sum_{n=0}^{N} \psi_{m n}(t) \psi_{m}(x) \psi_{n}(y) \\
T(x, y, t)=\sum_{m=0}^{M} \sum_{n=0}^{N} T_{m n}(t) \varphi_{m}(x) \varphi_{n}(y)+x
\end{array}\right\}
$$

where, $M$ and $N$ are the truncation numbers in the $x$ - and $y$ directions respectively, and $w_{m n}, \psi_{m n}$ and $T_{m n}$ are the coefficients of expansion. The collocation points $\left(x_{i}, y_{j}\right)$ are taken to be:

$$
\left.x_{i}=\cos \left[\pi\left(1-\frac{i}{M+2}\right)\right], y_{j}=\cos \left[\pi\left(1-\frac{j}{N+2}\right)\right]\right\}
$$

where, $i=1, \ldots, M+1$ and $j=1, \ldots, N+1$. In order to calculate the unsteady solutions, the Crank-Nicolson and Adams-Bashforth methods together with the function expansion (10) and the collocation methods are applied to Eqns. (2) to (4). Details of this method are available in Gottlieb and Orszag [38] and Mondal [6].

\subsection{Resistance coefficient}

The resistance coefficient $\lambda$ is used as the representative quantity of the flow state. It is also called the hydraulic 
resistance coefficient, and is generally used in fluids engineering, defined as:

$$
\frac{P_{1}^{*}-P_{2}^{*}}{\Delta z^{*}}=\frac{\lambda}{d_{h}^{*}} \frac{1}{2} \rho\left\langle w^{*}\right\rangle^{2}
$$

where quantities with an asterisk denote dimensional ones, \langle\rangle stands for the mean over the cross section of the duct and $d_{h}^{*}$ is the hydraulic diameter. The mean axial velocity $\left\langle w^{*}\right\rangle$ is calculated by:

$$
\left\langle w^{*}\right\rangle=\frac{v}{4 \sqrt{2 \delta} d} \int_{-1}^{1} d x \int_{-1}^{1} \bar{w}(x, y, t) d y
$$

Since $\frac{P_{1}^{*}-P_{2}^{*}}{\Delta z^{*}}=G, \lambda$ is related to the mean non-dimensional axial velocity $\langle w\rangle=\sqrt{2 \delta} d\left\langle w^{*}\right\rangle / v$ as:

$$
\lambda=\frac{16 \sqrt{2 \delta} D n}{3\langle w\rangle^{2}}
$$

where, $\langle w\rangle=\sqrt{2 \delta} d \frac{\left\langle w^{*}\right\rangle}{v}$.

\subsection{Nusselt number}

In curved duct, theoretically Nusselt number $(\mathrm{Nu})$ is defined as the proportion between the convective and the conductive heat transfer across the frontier. As the time independent solution and the time-dependent behaviors are calculated in several ways in the ducting system, the mathematical representation of the Nusselt number is revealed differently. The indicia of heat transmission, the Nusselt number, $N u$, for steady solution can be expressed as:

$$
\left.N u=-\frac{d^{*}}{\Delta T^{*}}\left|\frac{\partial T^{*}}{\partial x^{*}}\right|_{x=0}\right)
$$

Again the Nusselt number for the unsteady solution at the cooled wall $\left(N u_{c}\right)$ and the heated wall $\left(N u_{h}\right)$ can be written as follows:

$$
\begin{aligned}
& N u_{\tau_{c}}=\frac{1}{2} \int_{-1}^{1}\left\langle\left\langle\left.\frac{\partial T}{\partial x}\right|_{x=-1}\right\rangle\right) d y, \\
& N u_{\tau_{h}}=\frac{1}{2} \int_{-1}^{1}\left\langle\left\langle\left.\frac{\partial T}{\partial x}\right|_{x=1}\right\rangle\right) d y
\end{aligned}
$$

Here the average of the time interval $\tau$ is denoted by $\langle\langle\rangle\rangle$. For the periodic oscillation, usually $\tau$ is chosen as one period and if the oscillation is chaotic, $\tau$ is taken as a suitable time interval.

\section{RESULTS AND DISCUSSION}

We rotate the duct in the positive and negative direction and perform time-evolution of the resistance coefficient $\lambda$ for $D n=$ 2000, $G r=100$ and $\delta=0.5$. Positive rotation means that the rotational direction is the same as the main flow direction while negative rotation means the rotation is opposite to the main flow direction.

\subsection{Case I: Positive rotation}

Here, we present time-evolution of the unsteady solution for positive rotation $(0 \leq \mathrm{Tr} \leq 1000)$. Figure 2 (a) shows time-history of $\lambda$ for $T r=0$. It is found that the flow is chaotic at $T r=0$, which is well justified by drawing the phase space of the timeevolution result as shown in Figure 2(b). Figure 2(b) shows that the whole $\lambda-\gamma$ plane, where $\gamma=\iint \psi d x d y$, is covered with irregular line spectrum which shows that the flow presented in Figure 2(a) is chaotic. To see the pattern variation of secondary flows and temperature distribution, we obtain velocity contour (streamlines of secondary flow) and isotherm for $T r=0$ as shown in Figure 2(c), where we find that the unsteady flow is an asymmetric 4- to 8-vortex solution. Then we perform time-progression of $\lambda$ for $T r=100$ and $T r=250$ as shown in Figures 3(a) and 4(a), respectively. It is found that the flow oscillates irregularly both at $T r=100$ and $T r=250$ that means the flow is chaotic for $T r=100$ and $T r=250$, which is justified by tracing out phase spaces of the time change of $\lambda$ as shown in Figures 3(b) and 4(b), respectively. Then with a view to observe the vortex structure of secondary flows and isotherm for the chaotic oscillation, typical contours of velocity profile and isotherm are shown in Figures 3(c) and 4(c) for $T r=100$ and $T r=250$ respectively, where it is seen that the chaotic oscillation is an asymmetric 4- to 6-vortex solution. If $T r$ is increased a little, for example $\operatorname{Tr}=300$, it is found that the flow turns into multi-periodic and this multiperiodic oscillation prolongs for a small range of $\operatorname{Tr}(300 \leq \operatorname{Tr} \leq 320)$. Figures 5(a) and 6(a) show time progression results for $T r=300$ and $T r=320$ respectively, where multiperiodic solution is observed. These multi-periodic oscillations are well justified by depicting the phase spaces as shown in Figures 5(b) and 6(b) for $T r=300$ and $T r=320$, respectively. As seen in Figures 5(b) and 6(b), the line spectrum creates multiple orbits in its phases so that the unsteady flows presented in Figures 5(a) and 6(a) are multiperiodic. Contours of secondary velocity and isotherm are displayed in Figures 5(c) and 6(c) for $T r=300$ and $T r=320$ respectively. It is found that the flow oscillates multiperiodically in the asymmetric 4-vortex solution. If $T r$ is increased in the same direction a little further, it is found that the flow becomes periodic. The study shows that the transient flow is periodic for a small range of $\operatorname{Tr}(350 \leq \operatorname{Tr} \leq 370)$. Figure 7(a) shows time-progression result for $\operatorname{Tr}=350$ and its enlargement in Figure 7 (b) for $10.0 \leq t \leq 11.0$, which shows that the transient flow oscillates in a regular pattern that means the flow is periodic. This periodic oscillation is well vindicated by sketching the phase-space of the time evolution result as shown in Figure 7(c). As seen in Figure 7(c), the flow creates a single orbit in the $\lambda-\gamma$ plane so that the flow presented in Figure 7(a) is periodic. In fact, the periodic oscillation, which is detected in the present study, is a traveling-wave solution advancing in the downstream direction which is well-justified in the investigation by Yanase et al. [39] for three-dimensional (3D) travelling-wave solutions as an appearance of twodimensional (2D) periodic oscillation. Therefore, it is found that $2 \mathrm{D}$ calculations can accurately calculate the existence of $3 \mathrm{D}$ traveling-wave solutions by showing a presence of $2 \mathrm{D}$ periodic oscillation. To observe the formation of secondary vortices for the periodic oscillation, as time proceeds, typical contours of secondary velocity and isotherm are shown in Figure 7(d) for $T r=350$. It is found that flow oscillates in the asymmetric 4 -vortex solution. 


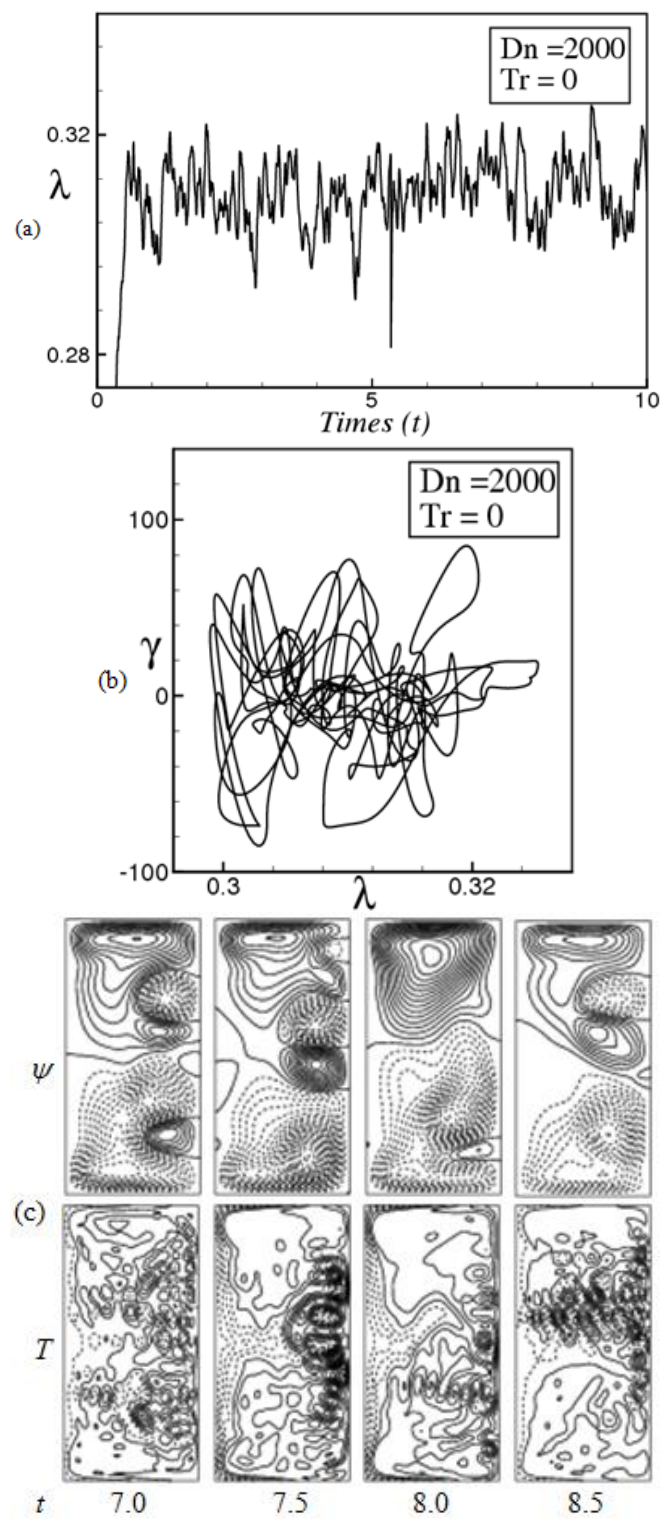

Figure 2. (a) Time-progression of $\lambda$, (b) Phase plot, (c) Seconary flow (top) and isotherm (bottom); for $T r=0$

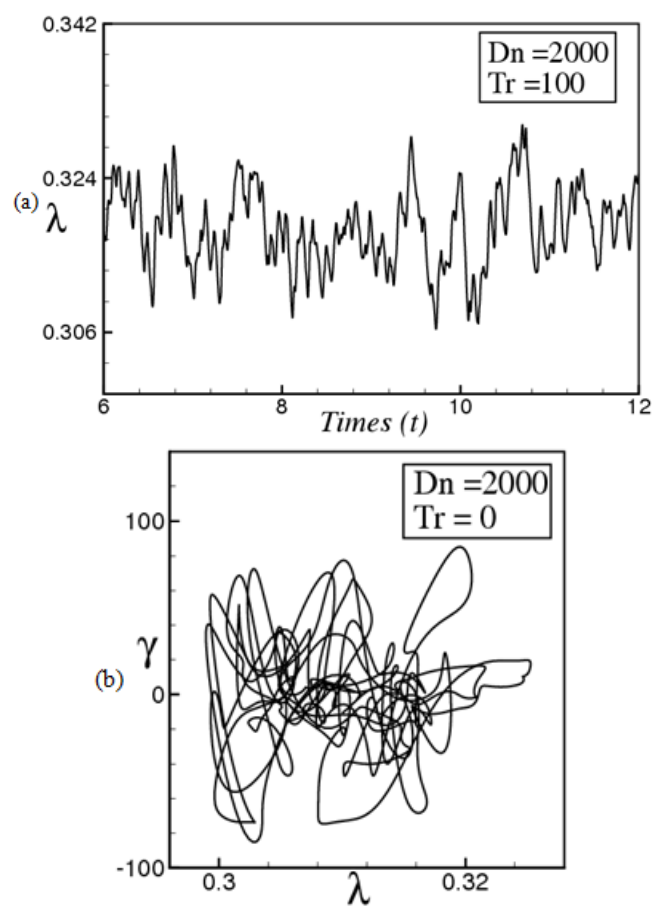

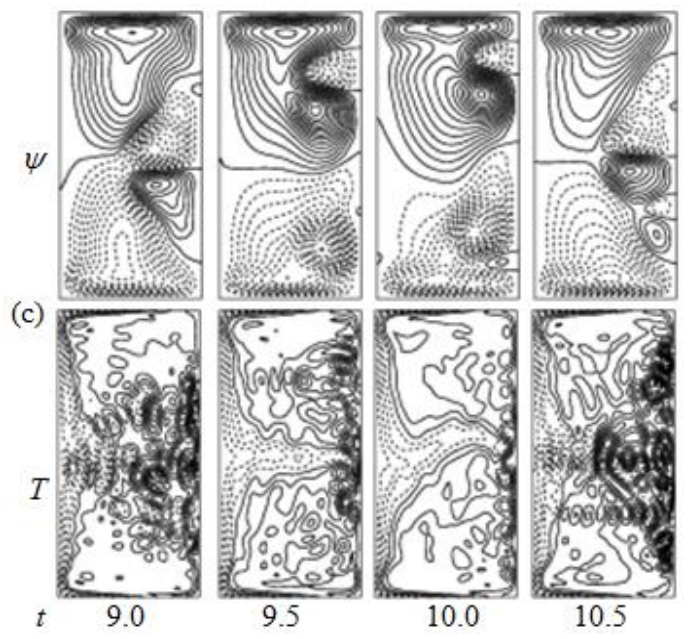

Figure 3. (a) Time-progression of $\lambda$, (b) Phase plot, (c) Seconary flow (top) and isotherm (bottom); for $T r=100$
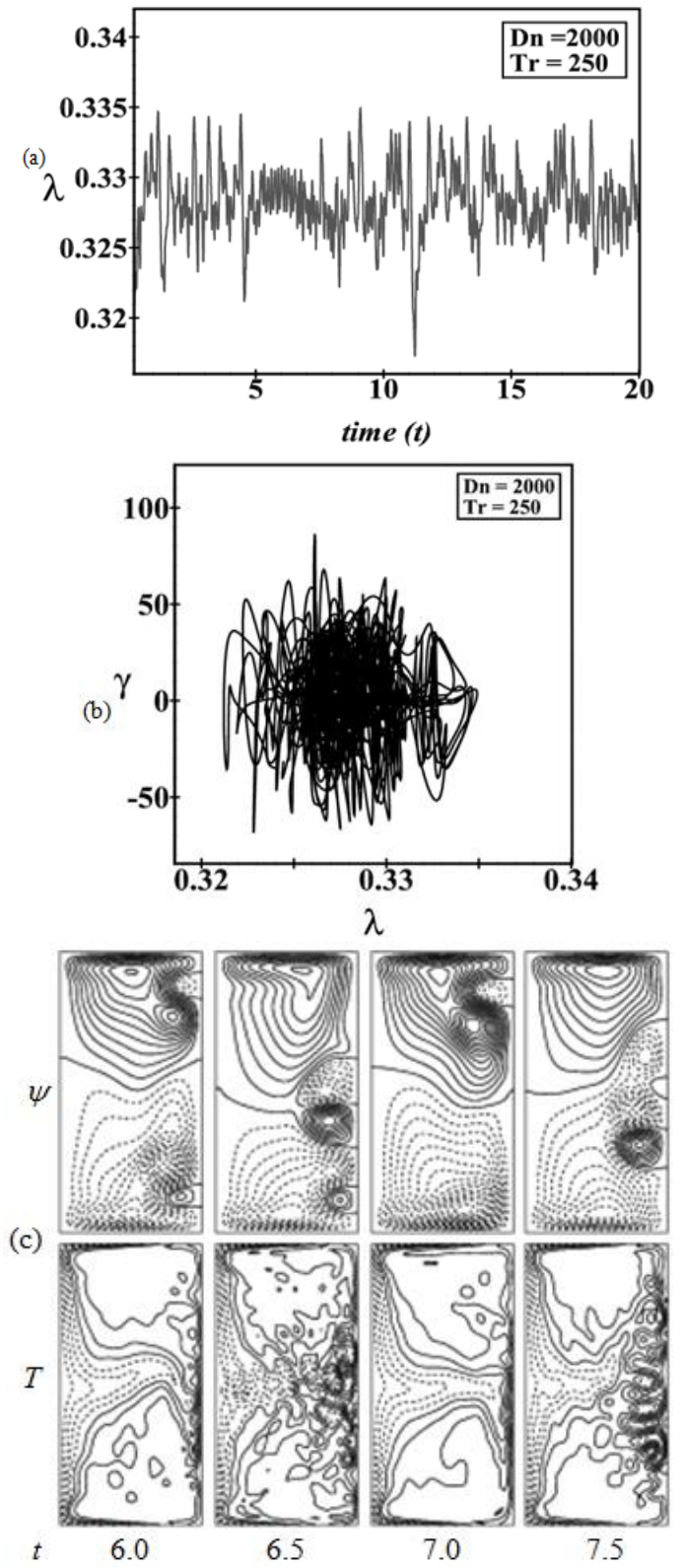

Figure 4. (a) Time-progression of $\lambda$, (b) Phase plot, (c) Seconary flow (top) and isotherm (bottom); for $\operatorname{Tr}=250$ 


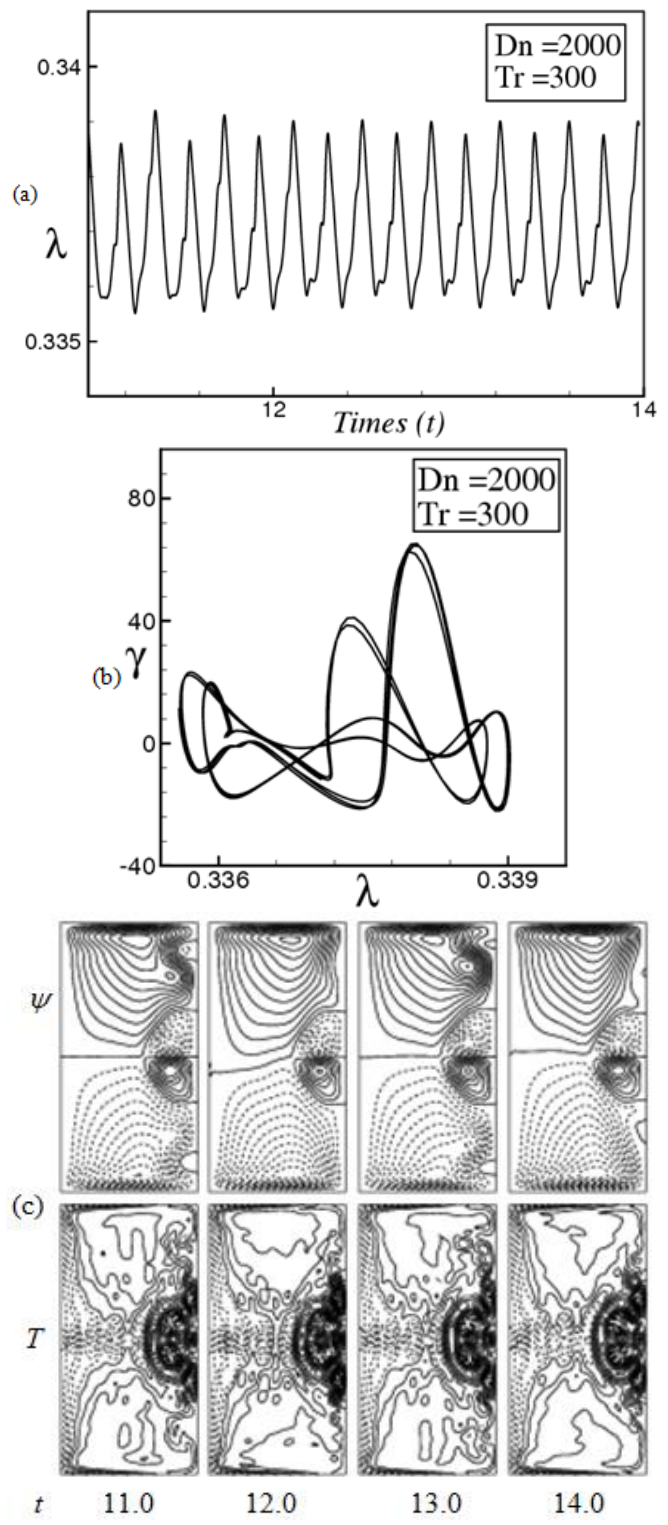

Figure 5. (a) Time-progression of $\lambda$, (b) Phase plot, (c) Seconary flow (top) and isotherm (bottom); for $T r=300$

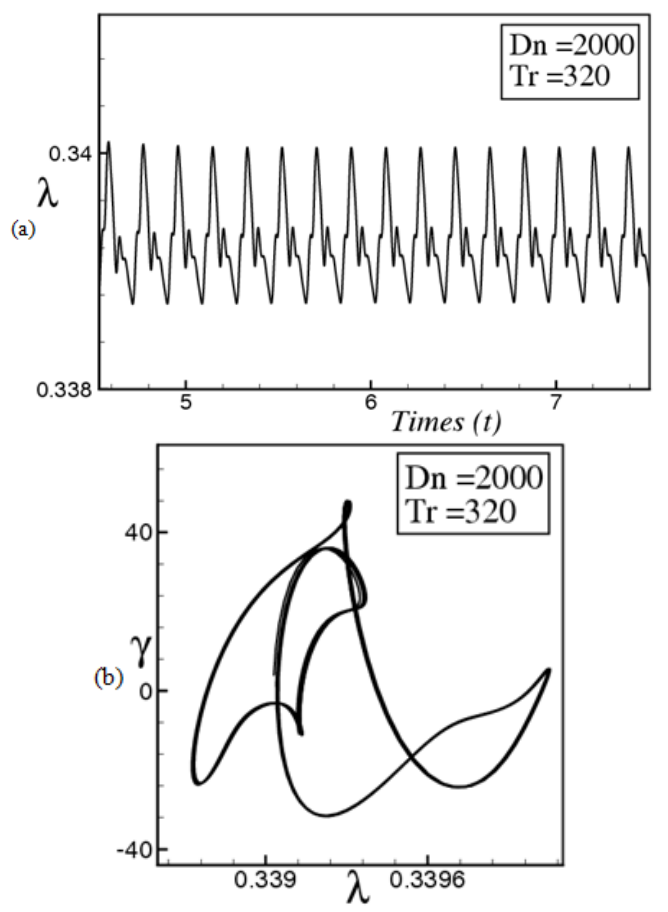

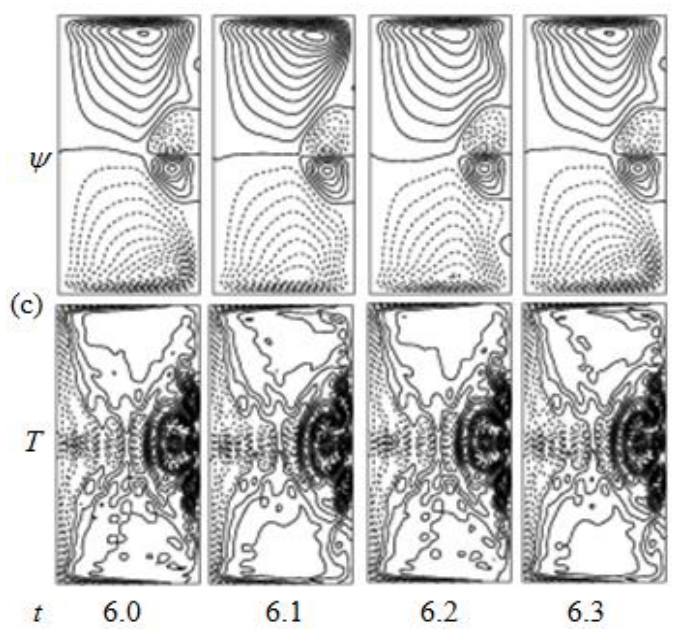

Figure 6. (a) Time-progression of $\lambda$, (b) Phase plot, (c) Seconary flow (top) and isotherm (bottom); for $\operatorname{Tr}=320$

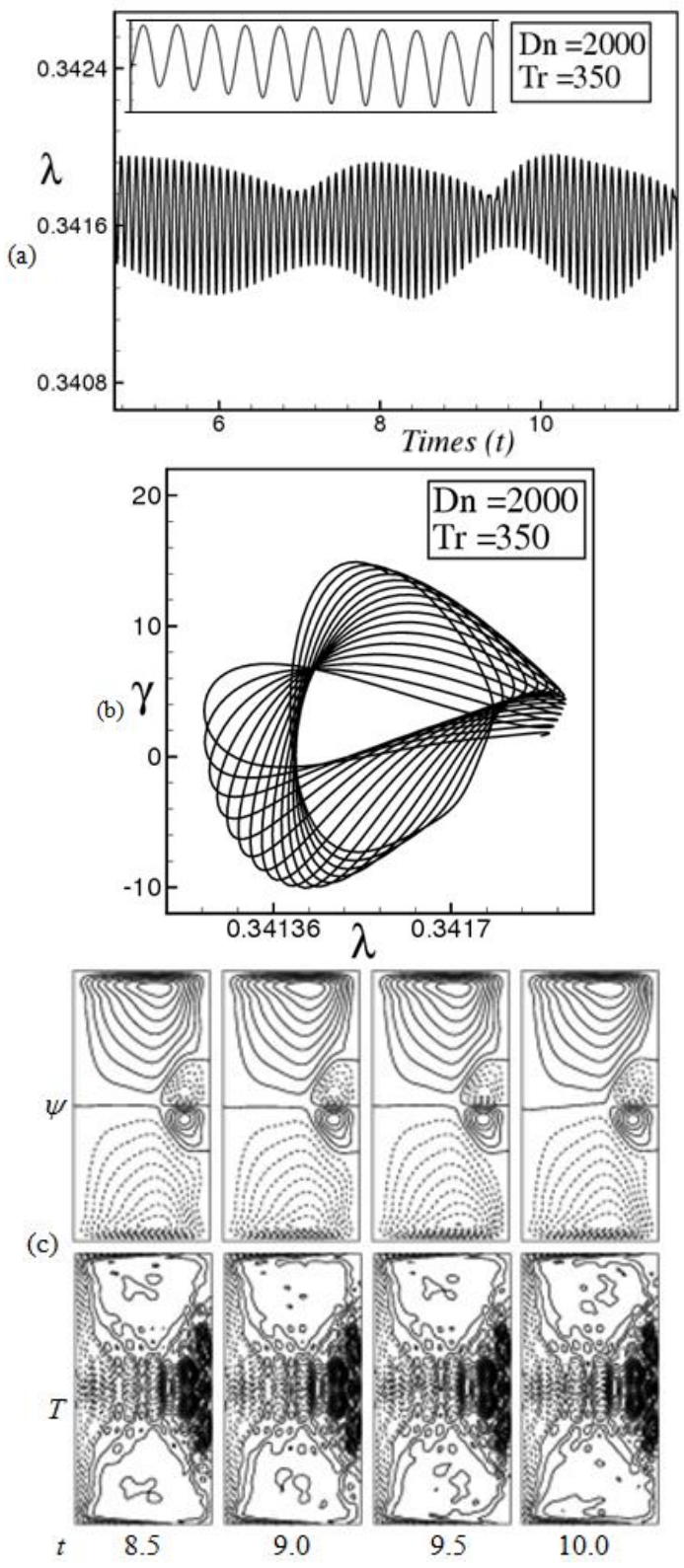

Figure 7. (a) Time-progression of $\lambda$, (b) Phase plot, (c) Seconary flow (top) and isotherm (bottom); for $\operatorname{Tr}=350$ 


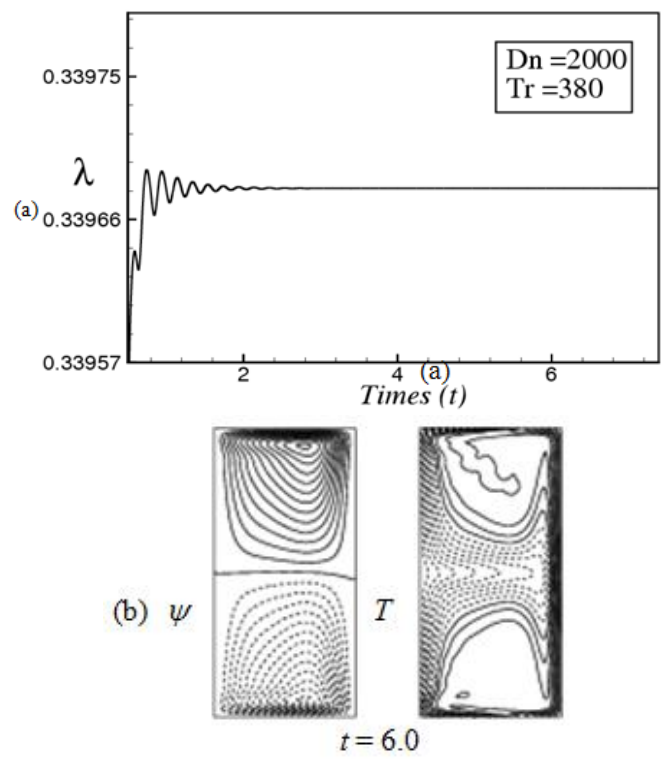

Figure 8. (a) Time-progression of $\lambda$, (b) Seconary flow (top) and isotherm (bottom); for $T r=380$

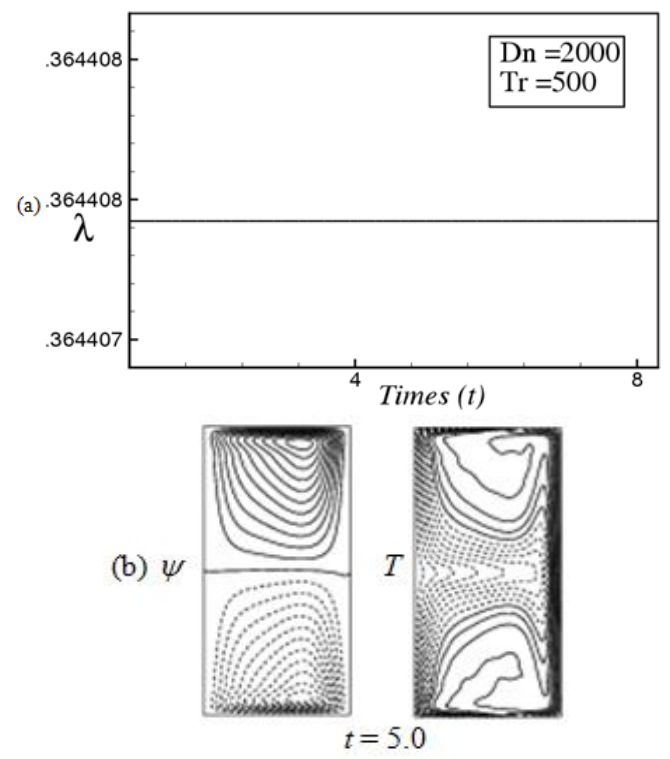

Figure 9. (a) Time-progression of $\lambda$, (b) Seconary flow (top) and isotherm (bottom); for $T r=500$

If $T r$ is increased further in the positive direction, we obtain steady-state solution as we predict the general scenario of fluid dynamics. Figures 8(a) and 9(a) show time evolution results for $\operatorname{Tr}=380$ and $\operatorname{Tr}=500$ respectively, and it is found that the unsteady flow is a steady-state solution for both the cases. Since the flow is steady-state, a single contour of fluid velocity and isotherm is shown in Figures 8(b) and 9(b) for $T r=380$ and $T r=500$ respectively, and it is found that the timedependent flows are asymmetric 2-vortex solutions for both the cases. The present study shows that temperature distribution is consistent with the secondary vortices, and secondary flow enhances heat transfer in the flow through vortex generation. In this study, it is found that combined action of the centrifugal, Coriolis and buoyancy forces aids to increase the number of secondary vortices, sometimes called Dean vortices, and as the flow becomes chaotic, the number of secondary vortices increases and consequently heat transfer is enhanced significantly from the heated wall to the fluid. In this regard, it should be noted that, the occurrence of the chaotic state, as presented in the present study, is related with destabilization of the periodic or quasi-periodic solutions which reminds us the case of Lorenz attractor [40]. It may be possible that the transition in the present study is caused by a similar mechanism as that of Ruelle-Takens scenario [41] in the laminar flow.

\subsection{Vortex structure for positive rotation}

In order to see the vortex-structure of secondary flows for positive rotation, streamlines of secondary flow and isotherms are predicted for the steady-state, periodic/multi-periodic and chaotic solutions for various values of $T r$ as shown in Figure 10. Figure 10 shows velocity contours and isotherm for the chaotic solutions at $T r=0,100$ and 250 , periodic or multiperiodic solutions at $\operatorname{Tr}=300,350$ and 370, and steady-state solutions at $\operatorname{Tr}=500$ and 1000, where 4- to 6-vortex solutions are obtained for the chaotic solution, 4-vortex for the periodic/multi-periodic solution while only 2-vortex for the steady-state solution. It is found that the number of secondary vortices increases as the flow becomes chaotic, and therefore it is suggested that chaotic solutions enhance heat transfer more efficiently than the steady-state or periodic solutions; this is because many secondary vortices are engendered at the outer concave wall of the duct [8] if the flow is chaotic. The periodic and chaotic behaviors are well discussed by Mondal et al. [42] and Mondal et al. [43]. Later, steady and unsteady behavior together with the flow velocity and the isotherms for the rotating and non-rotating curved duct is investigated by Nahid et al. [44], Nipa et al. [45], and Dolon et al. [46] study for square and rectangular rotating and non-rotating duct. They showed that though the unsteady behavior shows almost the same scenario such as steady-state, periodic, multi-periodic, and chaotic the flow characteristics are certainly influenced by the size, curvature, rotation of the duct. The irregular oscillations at $\mathrm{Tr}=0,100$ or 250 are termed as strong chaos [20]. The study shows that the number of secondary vortices is significantly increases for the chaotic solution at small rotation; however, as the rotation is increased gradually in the positive direction the chaotic flow turns into steady-state flow via multi-periodic and periodic flows, and consequently the number of secondary vortices decreases. Temperature profiles show that they are compatible with the secondary vortices, and a strong interaction is observed between the secondary flow pattern and temperature distribution.

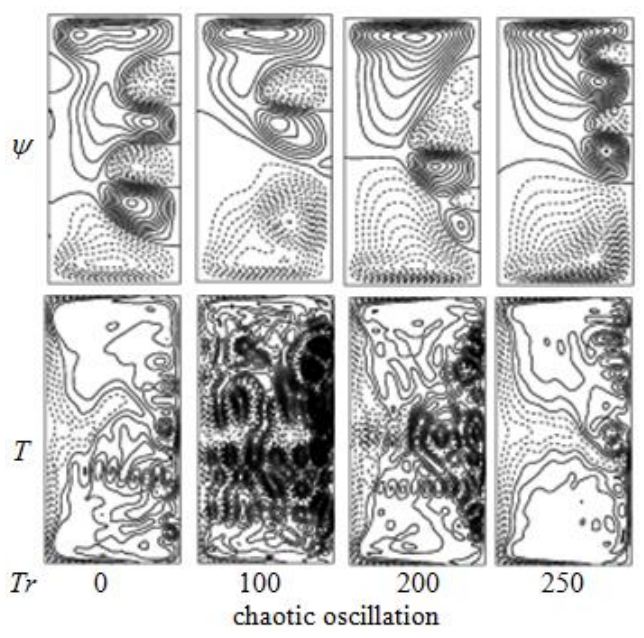




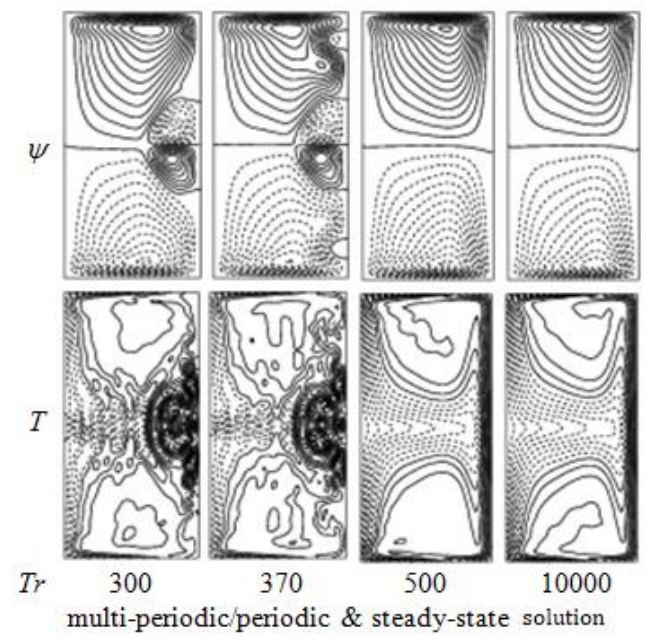

Figure 10. Vortex structure of secondary flows and isotherm for various values of $\mathrm{Tr}$

\subsection{Case II: Negative rotation}

Now, we performed time-history analysis for the negative rotation of the duct at $D n=2000$ and $\delta=0.5$. Figure 11 (a) shows time progression of $\lambda$ for $T r=-100$. It is found that the unsteady flow at $T r=-100$ is a chaotic oscillation, which is accurately justified by drawing the phase space as shown in Figure 11(b). As seen in Figure 11(b), the flow creates irregular orbits covering the $\lambda-\gamma$ plane so that the flow displayed in Figure 11(a) is chaotic at $\operatorname{Tr}=-100$. To observe the pattern variation of velocity profiles and isotherm, streamlines of secondary flow and isotherm are obtained as shown in Figure 11(c) for $T r=-100$, where it is found that the chaotic oscillation at $T r=-100$ is an asymmetric 4-vortex solution. Here we also observe that the streamlines of temperature profiles are consistence with the secondary vortices and convective heat generation is stronger in the whole position of the contour.
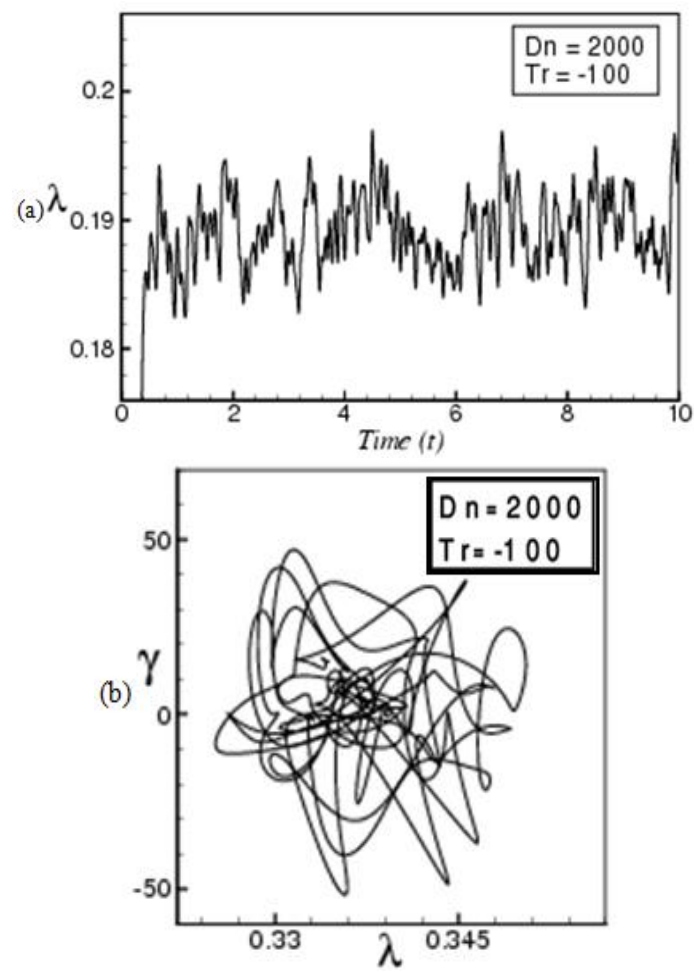

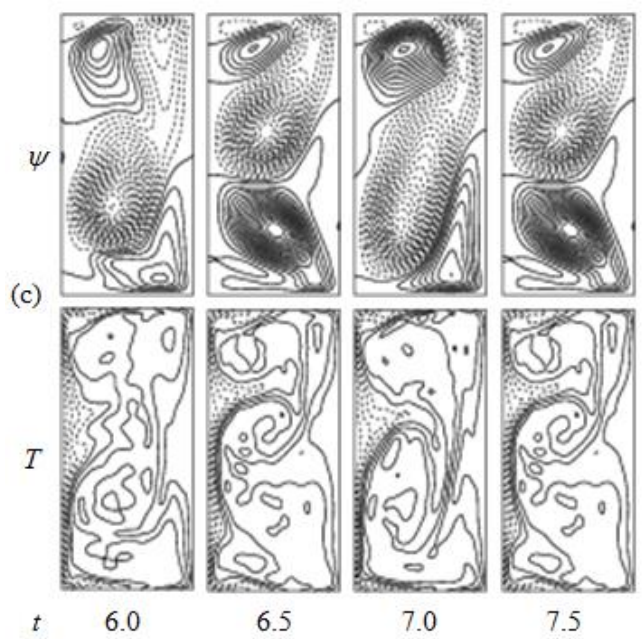

Figure 11. (a) Time-progression of $\lambda$, (b) Phase plot, (c) Seconary flow (top) and isotherm (bottom); for $T r=-100$

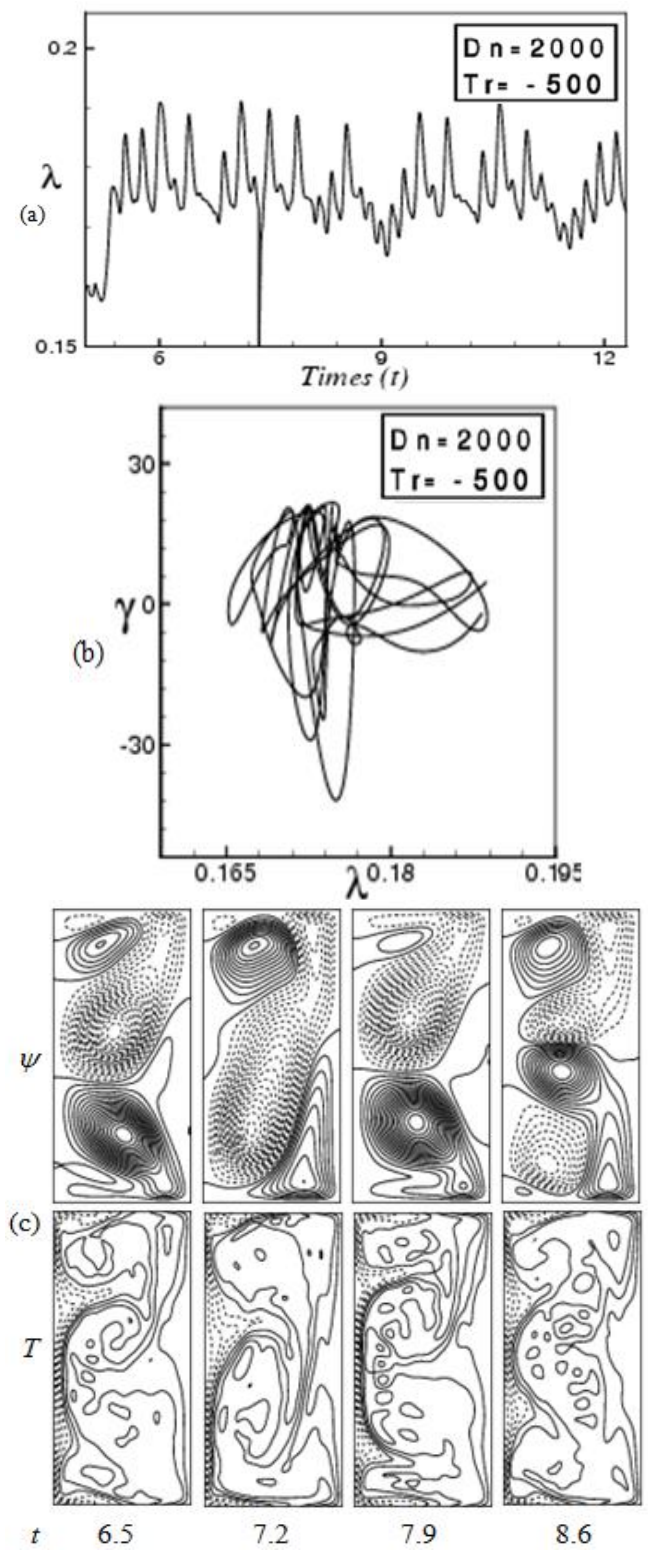

Figure 12. (a) Time-progression of $\lambda$, (b) Phase plot, (c) Seconary flow (top) and isotherm (bottom); for $T r=-500$ 

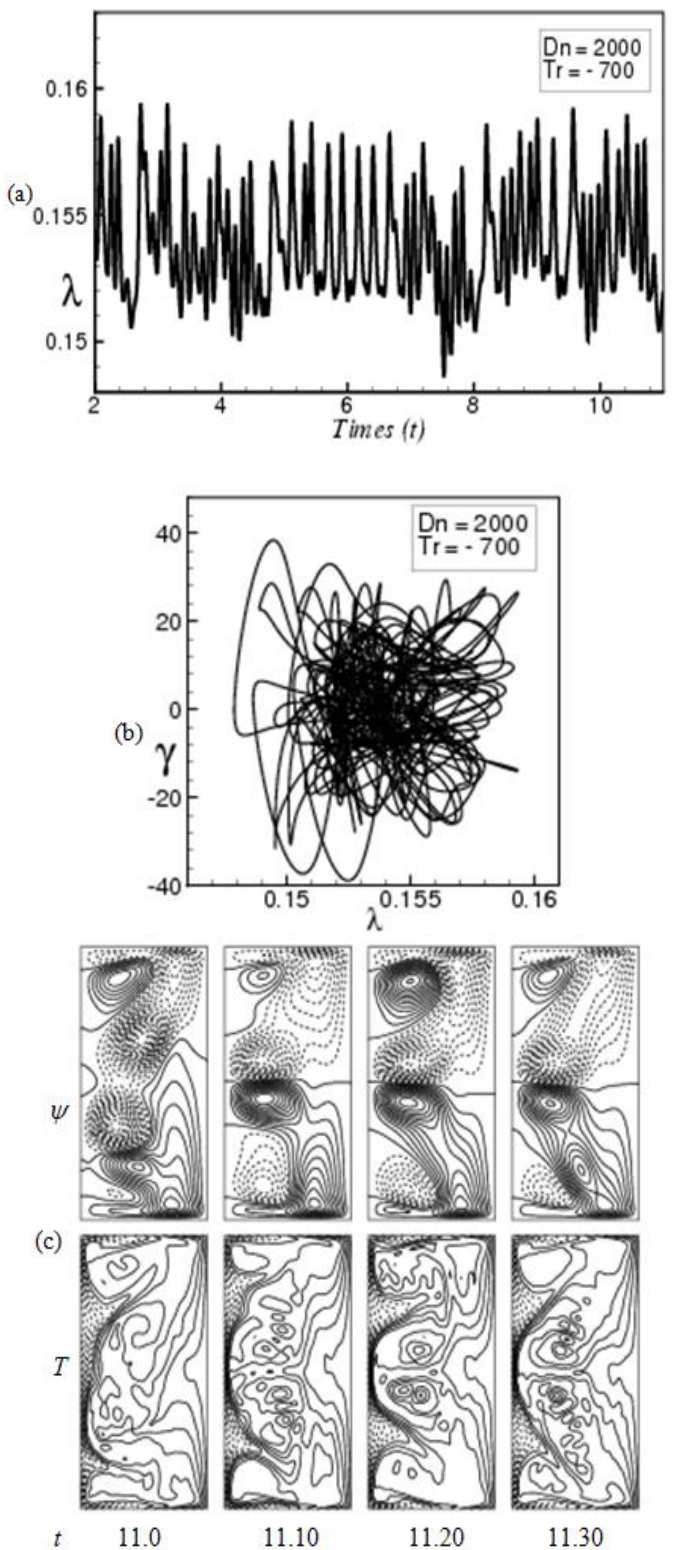

Figure 13. (a) Time-progression of $\lambda$, (b) Phase plot, (c) Seconary flow (top) and isotherm (bottom); for $T r=-700$

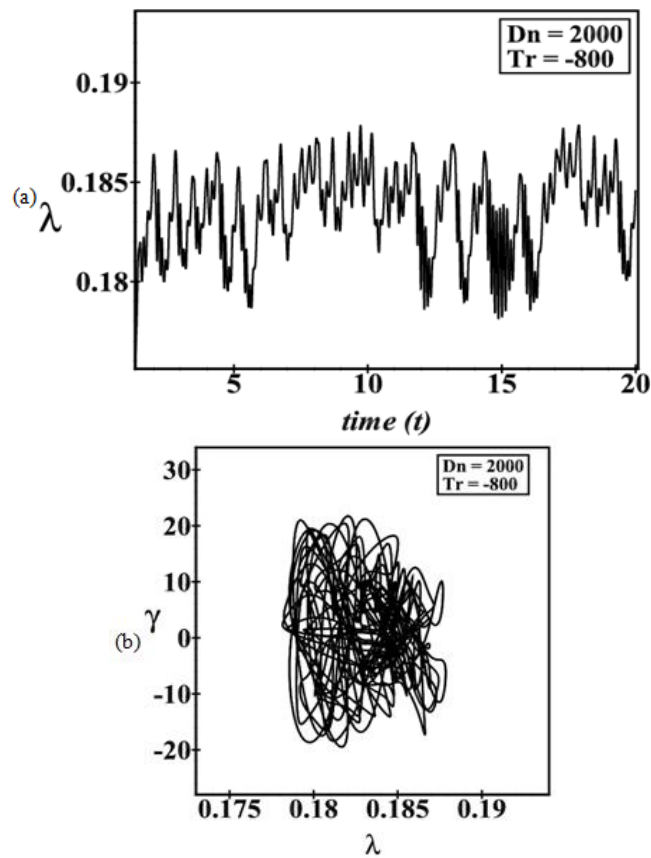

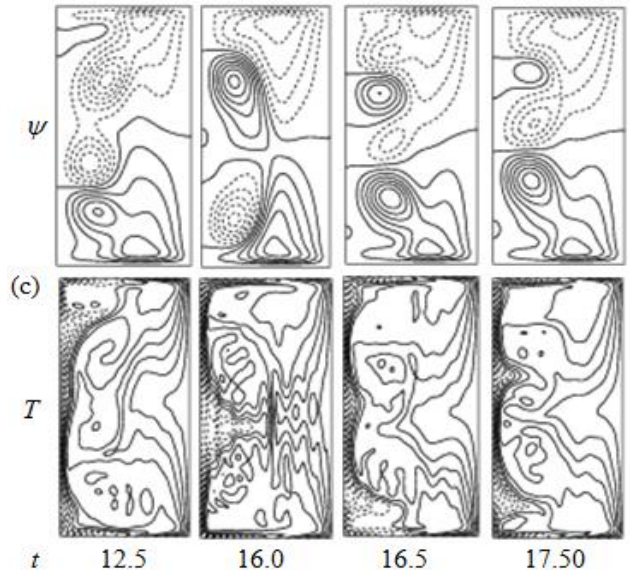

Figure 14. (a) Time-progression of $\lambda$, (b) Phase plot, (c) Seconary flow (top) and isotherm (bottom); for $T r=-800$
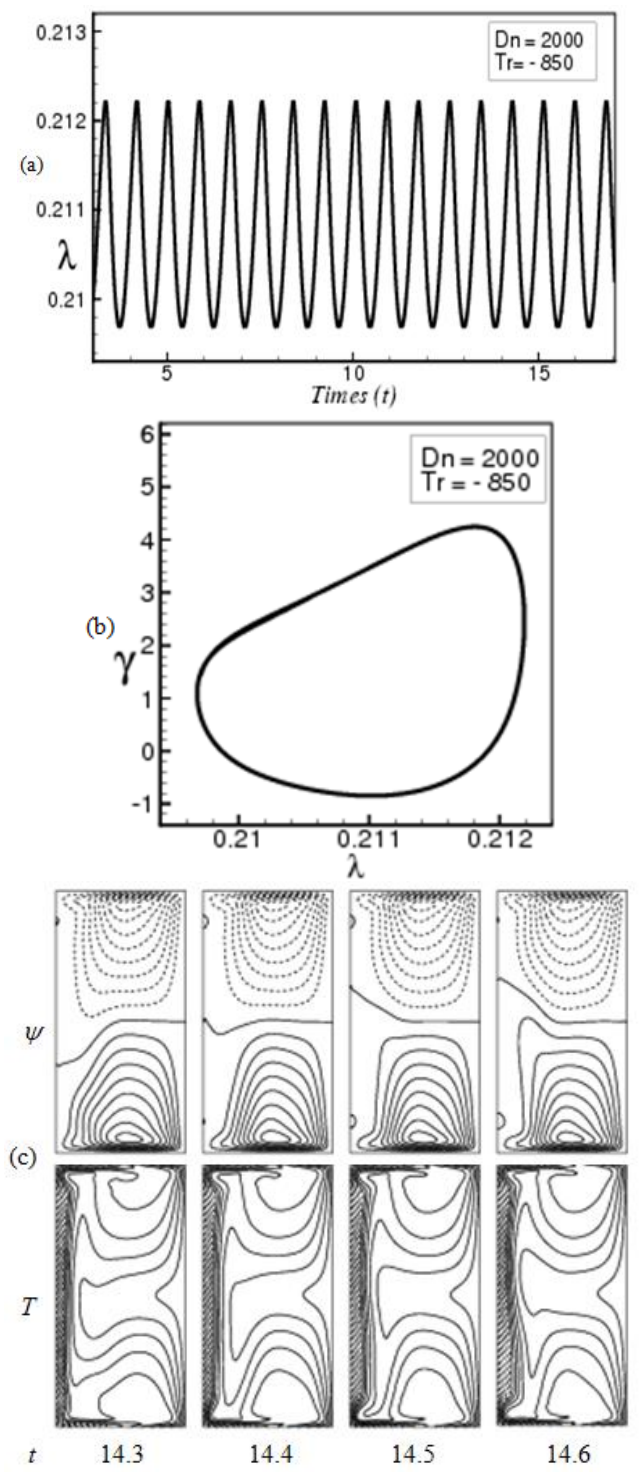

Figure 15. (a) Time-progression of $\lambda$, (b) Phase plot, (c) Seconary flow (top) and isotherm (bottom); for $\operatorname{Tr}=-850$

We continue this process and perform time dependent solution of $\lambda$ for $\operatorname{Tr}=-500$ and $\operatorname{Tr}=-700$ as shown in Figure 12(a) and 13(a), respectively. As seen in Figures 12(a) and 13(a), the time-dependent solutions at $\operatorname{Tr}=-500$ and $\operatorname{Tr}=-700$ are also chaotic. To observe the mode of oscillation of the 
chaotic oscillation, we draw the phase spaces of the time change of $\lambda$ for $T r=-500$ and $T r=-700$ as shown in Figures 12(b) and 13(b) respectively, and it is found that the transient solutions at $\operatorname{Tr}=-500$ and -700 are weak chaotic solution. Typical contours of secondary velocity and isotherms for $T r=$ -500 and $T r=-700$ are shown in Figures 12(c) and 13(c) respectively, where it is found that the chaotic oscillation at $T r$ $=-500$ and $\operatorname{Tr}=-700$ are asymmetric 4 - to 6 -vortex solution. Temperature profiles show that the streamlines of heat flow is uniformly distributed to all parts of the contour transferring heat from outer wall to the fluid, and the combined effects of rotation, pressure and buoyancy significantly change and increase the number of secondary vortices which play an important role in transferring heat from the heated wall to the fluid. Time advancement of $\lambda$ at $\operatorname{Tr}=-800$ is shown in Figure 14(a), which shows that the unsteady flow at $T r=-800$ is chaotic. This chaotic oscillation is well verified by drawing the phase space of the time advancement of $\lambda$ as shown in Figure 14(b). It is seen that the orbits of the flow-evolution intersect robustly which does not move in a regular pattern so that that the flow is chaotic at $\operatorname{Tr}=-800$. To see the pattern variation of velocity profiles, typical contours of secondary flow and isotherm for $\operatorname{Tr}=-800$ are attained as presented in Figure 14(c), and it is found that the chaotic flow oscillates in the asymmetric 4-vortex solution. If we accelerate the rotational speed in the negative direction at $T r=-850$, time progression result shows that the unsteady flow passes a regular pattern for a certain time interval that means the flow is periodic at $T r=$ -850 as shown in Figure 15(a). This periodic oscillation is well confirmed by drawing the phase space as shown in Figure 15(b). From Figure 15(b), it is visualized that the orbit of the flow-evolution does not intersect rather it moves in a regular pattern creating a single orbit, which signifies that the flow is time-periodic. Contours secondary velocity and isotherm for $\operatorname{Tr}=-850$ are then obtained as presented in Figure 15(c), and it is found that they oscillate between asymmetric 2-vortex solution. If we increase the simulation for strongly negative rotation of the duct, for example, $T r=-1000$ as shown in Figure 16(a), it is found that the periodic flow is changed into a steady-state nature, which creates asymmetric 2-vortex solution as shown in Figure 16(b). In this regard, it should be noted that Mondal et al. [47] executed spectral numerical study on unsteady fluid flow through a curved rectangular duct of small aspect ratio over a wide range of the Dean number $(D n)$ and the Grashof number $(G r)$. They obtained maximum 4vortex solution and mentioned that the unsteady flow becomes steady-state, periodic, multi-periodic or chaotic if $\mathrm{Dn}$ or $\mathrm{Gr}$ is increased. for in the ongoing study, the general scenario of the fluid dynamics which is- 'Chaotic $\rightarrow$ multiperiodic $\rightarrow$ periodic $\rightarrow$ steady-state' is confirmed for both positive and negative rotation of the duct.

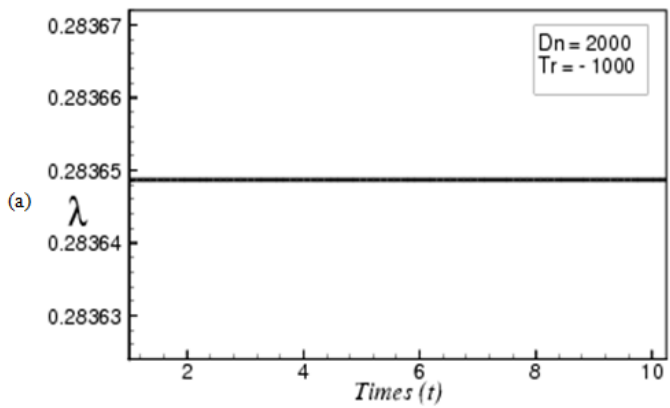

(b) $\psi$

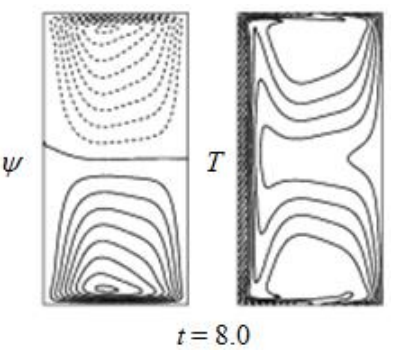

Figure 16. (a) Time-progression of $\lambda$, (b) Seconary flow (top) and isotherm (bottom); for $T r=-1000$

\subsection{Bar diagram of secondary flow structure}

Here, to observe the vortex-structure of secondary flows for the time-dependent solution at a glance, we draw a bar diagram to represent the formation of secondary vortices for timedependent solutions in the Taylor number vs. number of vortices $(\operatorname{Tr}-\theta)$ plane for both positive and negative rotation of the duct. Figure 17 shows vortex-structure of secondary flows on unsteady solutions for $\delta=0.5$. As seen in Figure 17, two- to multi-vortex solutions are produced at the same value of $T r$. It is found that maximum 8-vortex solution is attained at $\operatorname{Tr}=0$. However, as the rotation is imposed either in the positive or in the negative direction, for example when $T r=1000$ or $T r=$. 1000 , the number of secondary vortices declines gradually and finally we get only 2 -vortex solution. The reason is that strong centrifugal force and Coriolis force act in an opposite manner where buoyancy force is predominate and consequently number of secondary vortices decreases.

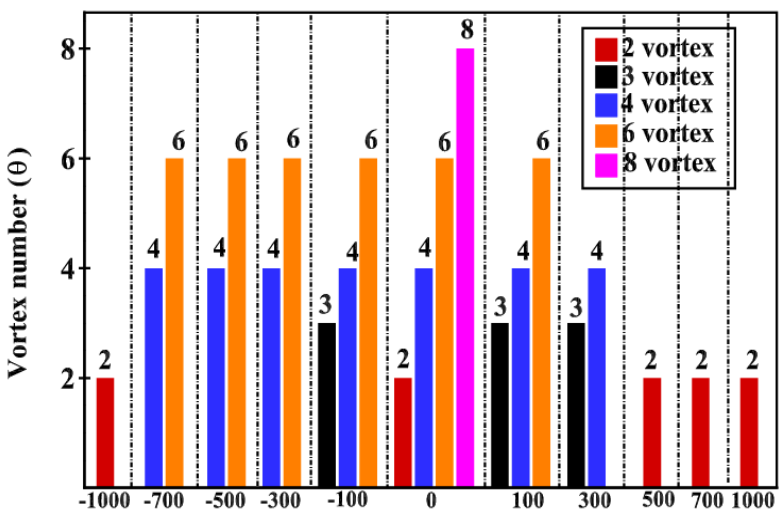

Taylor number $(\boldsymbol{T r})$

Figure 17. Vortex structure of secondary flows for various values of $\operatorname{Tr}$ at $\delta=0.5$ and $D n=2000$

\subsection{Convective heat transfer}

The Nusselt number $N u$, defined in Eq. (15), can be used as an index of horizontal heat transfer from the walls to the fluid. If the flow field is not steady, time-average of the Nusselt number, $N u_{\tau}$ is used as defined in Eq. (16). To study the convective heat transfer, variation of the steady values of $\mathrm{Nu}$ with $\operatorname{Tr}$ is shown in Figure 18 for both positive and negative rotation. Time-average of $N u$, calculated by the time-evolution computations on the inner and outer sidewalls for different solutions is also shown in the same figure. As seen in Figure 18(a) for positive rotation, $N u$ is larger at the heated wall than that at the cooling wall. It is also observed that $N u$ declines as $\mathrm{Tr}$ rises, and $\mathrm{Nu}$ for the cooling wall declines rapidly than the 
$N u$ for the heated wall. The reason is that the flow is chaotic at small $T r$ and as $T r$ increases the flow becomes steady-state. It is very interesting that, as seen in Figure 18(b) for the negative rotation, $N u$ for the heated wall increases rapidly for the heated wall while drops rapidly for the cooling wall as $T r$ increases in the negative direction. The reason is that, for the case of negative rotation heat transfer occurs substantially from the heated wall to the fluid than the cooling wall due to accumulating the significant number of secondary vortices at the outer concave wall.
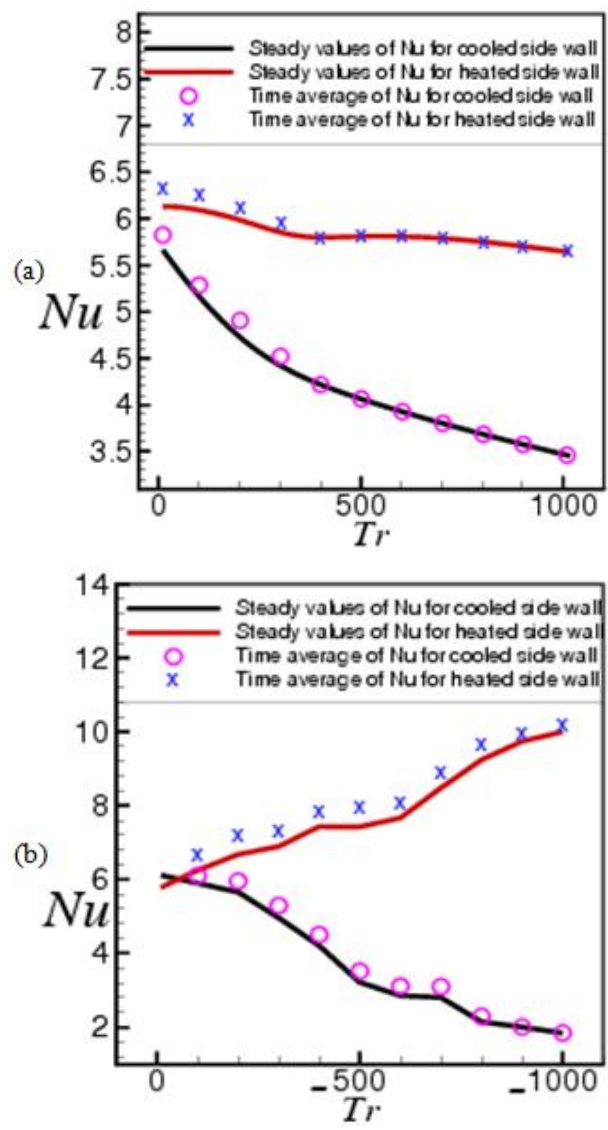

Figure 18. Variation of the Nusselt number $(N u)$ with the Taylor number $(T r)$; (a) Positive rotation, (b) Negative rotation

Then, to study convective heat transfer, temperature gradients (TG) on the cooling and heated sidewalls are calculated as shown in Figure 19 for positive rotation and in Figure 20 for negative rotation. As seen in Figures 19(a) and $20(\mathrm{a}), \frac{\partial T}{\partial x}$ on the cooling wall declines in the central region near $y=0$ as $T r$ increases. In the same figure, it is also seen that $\frac{\partial T}{\partial x}$ soar up in the regions other than the central region. This is due to advection of the secondary flow in the inward direction, which is a reverse flow of the outward secondary flow in the central region. As seen in Figures 19(b) and 20(b), $\frac{\partial T}{\partial x}$ raises monotonically over the whole region as $\operatorname{Tr}$ goes up from zero. It is observed that $\frac{\partial T}{\partial x}$ raises much higher at $T r=0$ or small values of $T r$ than that at large $T r$. Thus, as seen in Figures 19 and 20, the tendency of increasing TG at the heated wall is much significant than that in the cooling wall, which can be explained by the statement that heat transfer occurs considerably from the heated wall to the fluid and particularly at small $\operatorname{Tr}$ where the flow is chaotic.
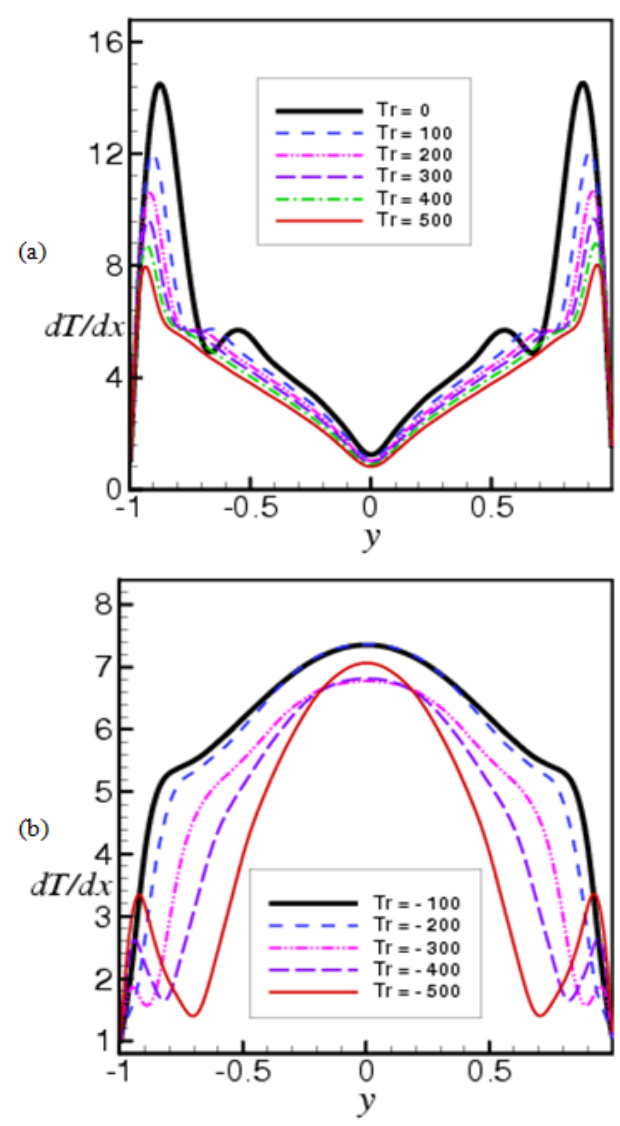

Figure 19. Temperature gradients for positive rotation; (a) cooled wall, (b) heated wall
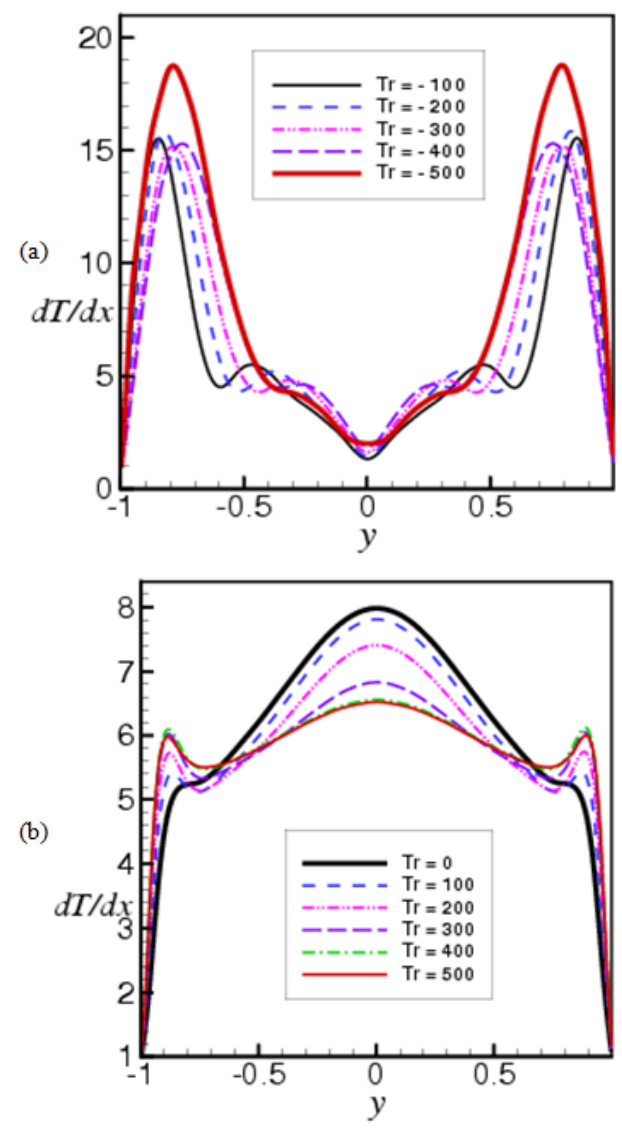

Figure 20. Temperature gradients for negative rotation; (a) cooled wall, (b) heated wall 


\subsection{Validation of the numerical result}

Here, we represent the validation of our computational results with the experimental studies performed by some authors. Figure 21 shows a comparative study of our computational result with the experimental investigation obtained by Chandratilleke [30] for the flow through a curved rectangular duct of aspect ratio 2 . We see that our computational results have a good agreement with the experimental data. Note that, till now no experimental studies have been found for rotating curved rectangular duct flow.

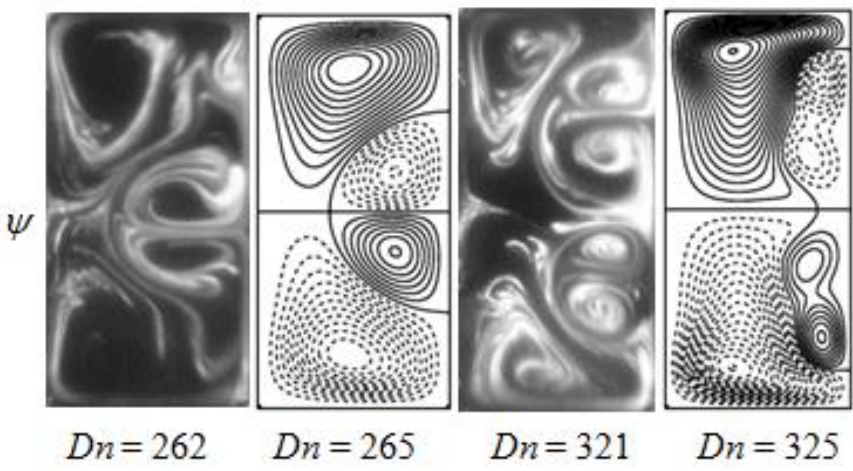

Figure 21. Experimental vs. numerical results. Left: experimental result by Chandratilleke [30] and right: numerical result by the authors

\section{CONCLUDING REMARKS}

Hydro-thermal behavior of transient fluid flow with natural and forced convective heat transfer through a rotating curved rectangular duct of aspect ratio 2 and curvature ratio 0.5 is investigated numerically with the aid of spectral technique considering a strong pressure gradient force in the axial direction. The outer wall of the duct is heated while the inner wall cooled, the top and bottom walls being thermally insulated. The system is rotated about the vertical axis in the positive and negative direction over a wide range of the Taylor number $(T r)$. The numerical results are validated with the experimental data and a good matching is observed. The following conclusions have been drawn from the present study;

- Time-history analysis as well as phase-space of the time progression results shows that the transient flow undergoes in the consequence 'chaotic $\rightarrow$ multiperiodic $\rightarrow$ periodic $\rightarrow$ steady-state', if $T r$ is increased either in the positive or in the negative direction.

- Flow regimes are identified for different types of physically realizable solutions which shows that for positive rotation, the transient flow is chaotic for $0 \leq \operatorname{Tr} \leq 250$, multi-periodic or periodic for $300 \leq \operatorname{Tr} \leq 370$ and steady-state for $380 \leq \operatorname{Tr} \leq 1000$; for negative rotation, however, the transient flow is chaotic for $-700 \leq \operatorname{Tr}<0$, periodic/multi-periodic for $900 \leq \operatorname{Tr} \leq-750$ and steady-state for $-1000 \leq \operatorname{Tr} \leq-900$.

- Vortex generation of secondary flows show that the transient flow is asymmetric 2- to 8-vortex for the chaotic solution, 4- to 6-vortex for the periodic/multiperiodic solution while only 2 -vortex for the steadystate solution. The number of secondary vortices is significantly higher for the chaotic solution, while few for the steady-state solution.
- To study convective heat transfer, it is found that for positive rotation, Nusselt numbers $(\mathrm{Nu})$ declines as $T r$ rises, and $N u$ for the cooling wall drops rapidly than the $N u$ for the heated wall. For the negative rotation, however, $\mathrm{Nu}$ for the heated wall upsurges rapidly for the heated wall but falls promptly for the cooling wall.

- The study shows that chaotic solutions boost heat transfer more effectively than the steady-state or other solutions due to significant number of Dean vortices at the outer concave wall. A strong interaction is observed between the heating-induced buoyancy force and centrifugal-Coriolis instability in the rotating curved passage that inspires fluid mixing and subsequently enhances heat transfer in the fluid at a great deal.

\section{REFERENCES}

[1] Dean, W.R., Hurst, J.M. (1959). Note on the motion of fluid in a curved pipe. Mathematika, 6(1): 77-85. https://doi.org/10.1112/S0025579300001947

[2] Berger, S.A., Talbot, L., Yao, L.S. (1983). Flow in curved pipes. Annual Review of Fluid Mechanics, 15(1): 461-512.

https://doi.org/10.1146/annurev.fl.15.010183.002333

[3] Nandakumar, K., Masliyah, J.H. (1986). Swirling flow and heat transfer in coiled and twisted pipes. Advanced Transport Process, 4: 49-112.

[4] Ito, H. (1987). Flow in curved pipes. JSME International Journal, 30(262): 543-552. https://doi.org/10.1299/jsme1987.30.543

[5] Yanase, S., Kaga, Y., Daikai, R. (2002). Laminar flow through a curved rectangular duct over a wide range of aspect ratio. Fluid Dynamics Research, 31(3): 151-183. https://doi.org/10.1016/S0169-5983(02)00103-X

[6] Mondal, R.N. (2006). Isothermal and non-isothermal flows through curved ducts with square and rectangular cross sections. Mechanical Engineering.

[7] Chandratilleke, T.T., Nursubyakto. (2003). Numerical prediction of secondary flow and convective heat transfer in externally heated curved rectangular ducts. International Journal of Thermal Science, 42(2): 187-198. https://doi.org/10.1016/S1290-0729(02)00018-2

[8] Ghia, K.N., Sokhey, J.S. (1977). Laminar incompressible viscous flow in curved ducts of rectangular cross-section. Trans. ASME I: Journal of Fluids Engineering, 99(4): 640-648. https://doi.org/10.1115/1.3448875

[9] Ishigaki, H. (1996). Laminar flow in rotating curved pipes. Journal of Fluid Mechanics, 329: 373-388. https://doi.org/10.1017/S0022112096008956

[10] Selmi, M., Nandakumar, K., Finlay, W.H. (1994). A bifurcation study of viscous flow through a rotating curved duct. Journal of Fluid Mechanics, 262: 353-375. https://doi.org/10.1017/S0022112094000534

[11] Wang, L.Q., Cheng, K.C. (1996). Flow transitions and combined free and forced convective heat transfer in rotating curved channels: The case of positive rotation. Physics of Fluids, 8(6): 1553-1573. https://doi.org/10.1063/1.868930

[12] Mondal, R.N., Watanabe, T., Hossain, M.A., Yanase, S. (2017). Vortex-structure and unsteady solutions with convective heat transfer trough a curved duct. Journal of 
Thermophysics and Heat Transfer, 31(1): 243-253. https://doi.org/10.2514/1.T4913

[13] Chandratilleke, T.T., Nadim, N., Narayanaswamy, R. (2012). Vortex structure-based analysis of laminar flow behavior and thermal characteristics in curved ducts. International Journal of Thermal Science, 59: 75-86. https://doi.org/10.1016/j.ijthermalsci.2012.04.014

[14] Norouzi, M., Kayhani, M.H., Nobari, M.R.H., Demneh, M.K. (2009). Convective heat transfer of viscoelastic flow in a curved duct. World Academy of Science, Engineering and Technology, 32: 327-333. https://doi.org/10.5281/zenodo.1081071

[15] Chandratilleke, T.T., Nadim, N., Narayanaswamy, R. (2013). Analysis of secondary flow instability and forced convection in fluid flow through rectangular and elliptical curved ducts. Heat Transfer Engineering, 34(14): https://doi.org/10.1080/01457632.2013.777249

[16] Hasan, M.S., Mondal, R.N., Kouchi, T., Yanase, S. (2019). Hydrodynamic instability with convective heat transfer through a curved channel with strong rotational speed. AIP Conference Proceedings, 2121(1): 030006. https://doi.org/10.1063/1.5115851

[17] Hasan, M.S., Islam, M.M., Ray, S.C., Mondal, R.N. (2019). Bifurcation structure and unsteady solutions through a curved square duct with bottom wall heating and cooling from the ceiling. AIP Conference $\begin{array}{lll}\text { Proceedings, } & \text { 2121(1): } & 050003 .\end{array}$ https://doi.org/10.1063/1.5115890

[18] Yanase, S., Nishiyama, K. (1988). On the bifurcation of laminar flows through a curved rectangular tube. Journal of Physical Society of Japan, 57(11): 3790-3795. https://doi.org/10.1143/JPSJ.57.3790

[19] Wang, L., Yang, T. (2005). Periodic oscillation in curved duct flows. Physica D, 200(3-4): 296-302. https://doi.org/10.1016/j.physd.2004.11.003

[20] Mondal, R.N., Kaga, Y., Hyakutake, T., Yanase, S. (2007). Bifurcation diagram for two- dimensional steady flow and unsteady solutions in a curved square duct. Fluid Dynamics Research, 39(5): 413-446. https://doi.org/10.1016/j.fluiddyn.2006.10.001

[21] Yamamoto, K., Xiaoyun, W., Kazuo, N., Yasutaka, H. (2006). Visualization of Taylor-Dean Flow in a curved duct of square cross-section. Fluid Dynamics Research, 38(1):

$1-18$. https://doi.org/10.1016/j.fluiddyn.2005.09.002

[22] Mondal, R.N., Kaga, Y., Hyakutake, T., Yanase, S. (2006). Effects of curvature and convective heat transfer in curved square duct flows. Trans. ASME, Journal of Fluids Engineering, 128(9): 1013-1022. https://doi.org/10.1115/1.2236131

[23] Mondal, R.N., Ray, S.C., Yanase, S. (2014). Combined effects of centrifugal and coriolis instability of the flow through a rotating curved duct with rectangular cross section. Open Journal of Fluid Dynamics, 4(1): 1-14. https://doi.org/10.4236/ojfd.2014.41001

[24] Razavi, S.E., Soltanipour, H., Choupani, P. (2015). Second law analysis of laminar forced convection in a rotating curved duct. Thermal Sciences, 19(1): 95-107. https://doi.org/10.2298/TSCI120606034R

[25] Krishna, C.V., Gundiah, N., Arakeri, J.H. (2017). Separation and secondary structures due to unsteady flow in a curved pipe. Journal of Fluid Mechanics, 815: 25-59. https://doi.org/10.1017/jfm.2017.7
[26] Islam, M.Z., Mondal, R.N., Rashidi, M.M. (2017). DeanTaylor flow with convective heat transfer through a coiled duct. Computers and Fluids, 149: 41-55. https://doi.org/10.1016/j.compfluid.2017.03.001

[27] Hasan, M.S., Mondal, R.N., Lorenzini, G. (2019). Numerical Prediction of non-isothermal flow with convective heat transfer through a rotating curved square channel with bottom wall heating and cooling from the ceiling. International Journal of Heat and Technology, 37(3): 710-726. https://doi.org/10.18280/ijht.370307

[28] Hasan, M.S., Islam, M.S., Badsha, M.F., Mondal, R.N., Lorenzini, G. (2020). Numerical investigation on the transition of fluid flow characteristics through a rotating curved duct. International Journal of Applied Mechanics and $\quad$ Engineering, 25(3): 45-63. https://doi.org/10.2478/ijame-2020-0034

[29] Hasan, M.S., Mondal, R.N., Lorenzini, G. (2020). Coriolis force effect in steady and unsteady flow characteristics with convective heat transfer through a curved square duct. International Journal of Mechanical Engineering, 5(1): 1-39.

[30] Chandratilleke, T.T. (2001). Secondary flow characteristics and convective heat transfer in a curved rectangular duct with external heating. 5th World Conference on Experimental Heat Transfer, Fluid Mechanics and Thermodynamics, Thessaloniki, Greece.

[31] Yanase, S. Mondal, R.N., Kaga, Y. (2005). Numerical study of non-isothermal flow with convective heat transfer in a curved rectangular duct. International Journal of Thermal Science, 44(11): 1047-1060. https://doi.org/10.1016/j.ijthermalsci.2005.03.013

[32] Norouzi, M., Kayhani, M.H., Nobari, M.R.H., Demneh, M.K. (2009). Convective heat transfer of viscoelastic flow in a curved duct. World Academy of Science, Engineering and Technology, 32: 327-333. https://doi.org/10.5281/zenodo.1081071

[33] Norouzi, M., Biglari, N. (2013). An analytical solution for dean flow in curved ducts with rectangular cross section. Physics of Fluids, 25(5): 053602. https://doi.org/10.1063/1.4803556

[34] Wu, X.Y., Lai, S.D., Yamamoto, K., Yanase, S. (2013). Numerical analysis of the flow in a curved duct. Advanced Materials Research, 706-708: 1450-1453. https://doi.org/10.4028/www.scientific.net/AMR.706708.1450

[35] Hasan, M.S., Mondal, R.N., Lorenzini, G. (2019). Centrifugal instability with convective heat transfer through a tightly coiled square duct. Mathematical Modelling of Engineering Problems, 6(3): 397-408. https://doi.org/10.18280/mmep.060311

[36] Hasan, M.S., Mondal, R.N., Lorenzini, G. (2020). Physics of bifurcation of the flow and heat transfer through a curved duct with natural and forced convection. Chinese Journal of Physics, 67: 428-457. https://doi.org/10.1016/j.cjph.2020.07.004

[37] Ray, S.C., Hasan, M.S., Mondal, R.N. (2020). On the onset of hydrodynamic instability with convective heat transfer through a rotating curved rectangular duct. Mathematical Modelling of Engineering Problems, 7(1): 31-44. https://doi.org/10.18280/mmep.070105

[38] Gottlieb, D., Orazag, S.A. (1977). Numerical analysis of spectral methods: Theory and applications. Society for Industrial and Applied Mathematics, Philadelphia. https://doi.org/10.1137/1.9781611970425 
[39] Yanase, S., Watanabe, T., Hyakutake, T. (2008). Traveling-wave solutions of the flow in a curved-square duct. Physics of Fluids, 20(124101): 1-8. https://doi.org/10.1063/1.3029703

[40] Lorenz, E.N. (1963). Deterministic nonperiodic flow. Journal of the Atmospheric Sciences, 20(2): 130-141. https://doi.org/10.1175/15200469(1963)020<0130:DNF>2.0.CO;2

[41] Ruelle, D. Takens, F. (1971). On the nature of turbulence. Communication in Mathematical Physics, 20: 167-192. https://doi.org/10.1007/BF01646553

[42] Mondal, R.N., Islam, M.S., Uddin, M.K., Hossain, M.A. (2013). Effects of aspect ratio on unsteady solutions through a curved duct flow. Applied Mathematics and Mechanics, 34(9):

$1-16$. https://link.springer.com/article/10.1007/s10483-0131731-8

[43] Mondal R.N, Islam M.Z, Islam M.S. (2013). Transient heat and fluid flow through a rotating curved rectangular duct: the case of positive and negative rotation. Procedia Engineering, 56 :

179-186. https://doi.org/10.1016/j.proeng.2013.03.105

[44] Islam, M.N., Ray, S.C., Hasan, M.S., Mondal, R.N. (2019). Pressure-driven flow instability with convective heat transfer through a rotating curved rectangular duct with differentially heated top and bottom walls. AIP Conference Proceedings, 2121(1): 030011. https://doi.org/10.1063/1.5115856

[45] Sultana, M.N., Hasan, M.S., Mondal, R.N. (2019). A numerical study of unsteady heat and fluid flow through a rotating curved channel with variable curvature. AIP Conference Proceedings, 2121(1): 030009. https://doi.org/10.1063/1.5115854

[46] Dolon, S.N., Hasan, M.S., Ray, S.C., Mondal, R.N. (2019). Vortex-structure of secondary flows with effects of strong curvature on unsteady solutions through a curved rectangular duct of large aspect ratio. AIP Conference Proceedings, 2121(1): 050004.

https://doi.org/10.1063/1.5115891

[47] Mondal, R.N., Islam, M.Z., Islam, M.M., Yanase, S. (2015). Numerical study of unsteady heat and fluid flow through a curved rectangular duct of small aspect ratio. Thammasat International Journal of Science and Technology, 20(4): 1-20. https://ph02.tcithaijo.org/index.php/SciTechAsia/article/ view/44164.

\section{NOMENCLATURE}

$\begin{array}{ll}D n & \text { Dean number } \\ G r & \text { Grashof number } \\ \mathrm{Pr} & \text { Prandtl number } \\ A & \text { Aspect ratio } \\ L & \text { Radius of the curvature } \\ X & \text { Horizontal axis } \\ Y & \text { Vertical axis } \\ Z & \text { Axis in the direction of the main flow } \\ U & \text { Velocity components in the } x \text {-direction } \\ V & \text { Velocity components in the } y \text {-direction } \\ W & \text { Velocity components in the } z \text {-direction } \\ T & \text { Temperature } \\ T & \text { Time }\end{array}$

\section{Greek symbols}

$\rho$

$\lambda$

$\mu$

$k$

$v$

$\psi$
Curvature of the duct

Density

Resistance coefficient

Viscosity

Thermal diffusivity

Kinematic viscosity

Sectional stream function 